\title{
Tomato (Solanum lycopersicum) SIIPT4, encoding an isopentenyltransferase, is involved in leaf senescence and lycopene biosynthesis during fruit ripening
}

\author{
Yong Zhang ${ }^{1}$, Zhengguo $\mathrm{Li}^{2}$, Yun Tu${ }^{1}$, Wenjing Cheng ${ }^{1}$ and Yingwu Yang ${ }^{1 *}$ (D)
}

\begin{abstract}
Background: Lycopene is an important carotenoid pigment in red fruits and vegetables, especially in tomato. Although lycopene biosynthesis and catabolism have been found to be regulated by multiple factors including phytohormones, little is known about their regulatory mechanism. Cytokinins are crucial to various aspects of plant growth. Isopentenyltransferases (IPTs) catalyze the initial rate-limiting step of cytokinins biosynthesis, however, their roles in fruit ripening remain unclear.

Results: Here, the functions of SIIPT4, encoding an isopentenyltransferase, were characterized via RNAi-mediated gene silencing in tomato. As we expected, silencing of SIIPT4 expression resulted in accelerated leaf senescence. However, down-expression of SIIPT4 generated never-red orange fruits, corresponding with a dramatic reduction of lycopene. Among lycopene biosynthesis-related genes, the fact of remarkable decrease of ZISO transcript and upregulation of other genes, revealed that SIIPT4 regulates positively lycopene biosynthesis via directly affecting ZISO expression, and also supported the existence of regulatory loops in lycopene biosynthesis pathway. Meanwhile, the accumulation of abscisic acid (ABA) was reduced and the transcripts PSY1 were increased in SIIPT4-RNAi fruits, supporting the feedback regulation between $A B A$ and lycopene biosynthesis.
\end{abstract}

Conclusion: The study revealed the crucial roles of SIIPT4 in leaf senescence and the regulatory network of lycopene biosynthesis in tomato, providing a new light on the lycopene biosynthesis and fruit ripening.

Keywords: Carotenoids, Cytokinins, Fruit ripening, Leaf senescence, Lycopene, SIIPT4, Tomato

\section{Background}

Carotenoids are a group of terpenoid pigments, naturally synthesized in plants, fungi, algae and photosynthetic bacteria [1], and usually give bright colors to fruit, flower and seed in plants $[2,3]$. Carotenoids are more than just pigments, they also act as membrane stabilizers and the precursors of important plant hormones, such as abscisic acid (ABA) and strigolactone, to play important roles in photosynthesis and a variety of physiological processes including plant growth, fruit development and response to abiotic stress [4-6]. Lycopene is a bright red linear carotenoid and widely exists in red fruits and vegetables.

\footnotetext{
* Correspondence: yangyinwu@cqu.edu.cn

'Bioengineering College, Chongqing University, Chongqing 400044, China Full list of author information is available at the end of the article
}

Moreover, Lycopene is of particular nutritional interest in promoting health and reducing the risk of various diseases, especially cancer and cardiovascular disease [7-9].

In recent years, significant progress has been achieved in understanding of carotenoid biosynthesis and catabolism using biochemical and genetics approaches [6]. Lycopene is produced from carotenoid biosynthesis pathway, and has been proposed to proceed through a poly-cis pathway: geranylgeranyl diphosphate (GGPP) $\rightarrow$ 15-cis-phytoene $\rightarrow$

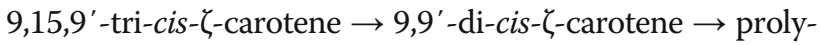
copene $\rightarrow$ all-trans-lycopene, catalyzed by phytoene syn-

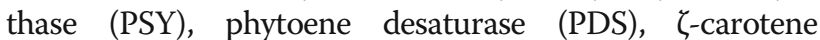
isomerase (ZISO), $\zeta$-carotene desaturase (ZDS), and carotene isomerase (CrtISO), respectively [2, 3, 10-12]. Lycopene can be further cyclized to produce carotenoids with 
two $\beta$ rings ( $\beta$-carotene) and one $\beta$ ring and one $\varepsilon$ ring ( $\alpha$-carotene). In higher plants, lycopene- $\beta$-cyclase (LCYB) and lycopene $\varepsilon$-cyclase (LCYE) catalyze the formation of $\beta$-rings and $\varepsilon$-rings, respectively [13, 14]. The $\alpha$-carotene and $\beta$-carotene can be hydroxylated to generate xanthophyll pigments lutein and zeaxanthin, respectively. Zeaxanthin is the precursor of apocarotenoid hormone ABA [3]. Plant carotenoid biosynthesis and catabolism have been found to be regulated by developmental programs, environmental factors, metabolic signals, and classic phytohormones including ethylene, auxin, and ABA $[2,6]$.

Tomato (Solanum lycopersicum) is by far the largest dietary source of lycopene [9], and also a model system for fleshy fruit development and ripening, exhibiting active cell division and expansion at the early developmental stages, and dramatic changes in texture and carotenoid, sugar, and acid content during ripening stages [15]. In tomato fruit, lycopene is the predominant carotenoid pigment and principally responsible for the deep-red color, which is the most obvious characteristic of ripe fruit [16]. For lycopene accumulation during tomato fruit ripening, the transcripts of genes modulating lycopene biosynthesis are upregulated, on the contrary, the expression of genes encoding the enzymes that metabolize lycopene are dramatically decreased [17]. Two tomato mutants altering lycopene content have been characterized: yellow-flesh (locus $r$ ) exhibits yellow fruits as a result of a loss-of-function mutant of the PSY1 gene [18], and tangerine mutant (locus $t$ ) appears orange fruits due to a mutation in the $\mathrm{CrtISO}$ gene [19]. Additionally, several transcription factors and genes are involved in the regulatory network of carotenoid biosynthesis. The ripening inhibitor (RIN), a MADS box transcription factor, is able to affect the accumulation of lycopene by directly interacting with the promoter of $P S Y 1$ gene [20]. A STAY-GREEN protein SGR1 can regulate lycopene accumulation through directly interacting with PSY1 to inhibit its activity during tomato fruit maturation [21]. RNAi-mediated silencing of $A P 2 a$, encoding a tomato APETALA2/Ethylene Responsive Factor (AP2/ERF) transcription factor, reduced total carotenoids accumulation and downregulated the expression of PSY1 [22]. In Arabidopsis, the AtAP2 and phytochrome-interacting factor 1 (AtPIF1) regulate carotenoid biosynthesis by directly binding to the promoter of AtPSY1 gene [23, 24]. Although these genes were reported to regulate carotenoid biosynthesis, they focused mainly on repressing the PSY1 expression in plants. The knowledge on the exact regulation mechanism of carotenoid/lycopene biosynthesis in response to various developmental and environmental factors and so on is still limit so far.

The phytohormone cytokinins (CKs) play crucial roles in a wide aspects of plant growth and development, including cell division, fruit development, leaf senescence, apical dominance, lateral root formation, and stress tolerance
[25-28]. Throughout fruit ontogeny, it is known that CKs and their biosynthesis-related genes may play important roles in fruit set and development, however, to our knowledge, little is known about their impact on fruit ripening. The adenosine phosphate-isopentenyltransferases (IPTs) catalyze the initial step of CK biosynthesis to produce isopentenyladenine (ip) nucleotides as CK precursors, it is the rate-limiting biosynthetic step of CKs [29, 30]. Plant IPTs belong to multigenic family and have been identified in Arabidopsis (AtIPT1-AtIPT9) [30] and tomato (SIIPT1-SIIPT6) [27]. Up to now, about the physiological significance of the six tomato SIIPTs, only SIIPT3 and SIIPT4 were reported to mediate salt stress response in tomato [31]. However, the authors only obtained the SIIPT3 overexpression tomato lines, and didn't generate any transgenic tomato plants of SlIPT4 overexpression or knockdown. So the physiological function of tomato SIIPT4 is still unknown.

In the present study, we report that the tomato ISOPENTENYLTRANSFERASE4 gene (SIIPT4, GenBank accession number AB690814), is involved in leaf senescence and pigment formation of ripe fruit. RNAi-mediated silencing of SIIPT4 accelerated leaf senescence and generated orange fruits with a significant reduction of lycopene content. The dramatic reduction of ZISO transcripts, moderate decrease of $Z D S$ mRNAs, and upregulation of other genes (PSY1, PDS, CrtISOs) in SIIPT4-silenced fruits, suggest that SIIPT4 controls lycopene biosynthesis via directly affecting ZISO expression, and also support the existence of regulatory loops in the carotenoids biosynthesis pathway. These data uncover that SIIPT4 is involved in the regulatory network of lycopene biosynthesis and plays a crucial role in color formation during fruit ripening in tomato.

\section{Methods \\ Plant materials and growth condition}

Tomato (Solanum lycopersicum cv. MicroTom) plants were grown under the following conditions: $14 / 10 \mathrm{~h}$ day/ night cycle, $25{ }^{\circ} \mathrm{C} / 20{ }^{\circ} \mathrm{C}$ day/night temperature, $80 \%$ humidity, and $250 \mu \mathrm{mol} \mathrm{m} \mathrm{m}^{-2} \mathrm{~s}^{-1}$ light intensity. For analyzing the organ-specific expression profiling of SIIPT4, the roots, stems, leaves, flowers, and fruits (mixture of mature green stage) were collected from 8-week-old wild-type tomato plants. Samples taken from the different parts (ovary, stamen, petal, and sepal) of flowers were harvested at bud ( $-2 \mathrm{dpa}$, day post anthesis), anthesis (0 $\mathrm{dpa})$, and post-anthesis (4 dpa) stages, respectively. The developmental stages of fruits investigated in this study were early mature green ( $25 \mathrm{dpa})$, mature green (35 dpa), break ( $\mathrm{Br}$, the first visible sign of carotenoid accumulation is evident in surface, $40 \mathrm{dpa})$, orange $(\mathrm{Br}+3$ days $)$, and ripening $(\mathrm{Br}+7$ days $)$. 


\section{Quantitative real-time PCR}

Total RNA samples were extracted using Trizol reagent (Invitrogen, USA) according to the manufacturer's instructions. The first-strand cDNA synthesis was performed using $1 \mu \mathrm{g}$ of total RNA by PrimeScript ${ }^{\mathrm{tw}}$ RT reagent Kit with gDNA Eraser (Takara, Japan). Quantitative real-time PCR (qRT-PCR) was performed using cDNAs corresponding to $2 \mathrm{ng}$ of total RNA in $10 \mu \mathrm{L}$ reaction volume using SYBR GREEN PCR Master Mix on a CFX96 Touch $^{\text {tw }}$ Real-Time PCR Detection System (Bio-Rad, USA). The qRT-PCR reactions were performed as follow: $95^{\circ} \mathrm{C}$ for $2 \mathrm{~min}$, followed by 40 cycle of $95{ }^{\circ} \mathrm{C}$ for $15 \mathrm{~s}$ and $58^{\circ} \mathrm{C}$ for $40 \mathrm{~s}$ and one cycle of $95^{\circ} \mathrm{C}$ for $15 \mathrm{~s}$ and $60{ }^{\circ} \mathrm{C}$ for $15 \mathrm{~s}$. The $2^{-\Delta \Delta \mathrm{Ct}}$ method was used for the analysis of relative gene expression levels as described by Yang et al. [32]. For all qRT-PCR experiments, at least three biological replicates were performed and each reaction was run in triplicate. Tomato Slactin-51 (GenBank accession number Q96483) was used as the reference gene [32]. The sequences of gene primers for qRT-PCR were listed in Additional file 1: Table S1.

\section{RNA interference (RNAi) vector construction and plant transformation}

A $340 \mathrm{bp}$ specific fragment of SIIPT4 was amplified from tomato cDNA using the following primers: $\mathrm{F}$ 5'-GGGGTACCAAGCTTTGCTGAATTGTCAAATTC CGTGG-3' with Kpn I and Hind III restriction sites, and $\mathrm{R}$ 5'-CCGCTCGAGTCTAGAATAGTGAGATGC TGCTGCCA-3' with Xho I and Xba I restriction sites. The PCR products were cloned into the pHANNIBAL vector in the sense orientation and anti-sense orientation into the Hind III-Xba I polylinker and the Kpn I-Xho I polylinker, respectively. Then the intron-spliced hairpin construct of SIIPT4 specific fragment under the transcriptional control of constitutive CaMV35S promoter and OCS terminator was subcloned as Spe I-Sac I fragment into pCAMBIA1301 binary vector, in which the hygromycin resistance gene has been replaced by the neomycin phosphotransferase II (nptII) gene. Transgenic plants were generated by Agrobacterium tumefaciens-mediated transformation. The positive transgenic lines were checked by histochemical $\beta$-glucuronidase (GUS) straining and PCR, and the silencing efficiency of SIIPT4 gene were detected by qRT-PCR.

\section{Drought treatment}

The 4-week-old wild-type tomato plants were stopped watering. When the leaves showed serious wilting, the leaves of drought stress plants and control plants supplied water normally were collected, respectively. Subsequently, the drought treatment plants were supplied water again, and the leaves of 2, 6, 12 and $24 \mathrm{~h}$ after watering were collected during the recovery process. The treatments were performed with three biological replicates.
Chlorophyll and carotenoids measurements

Chlorophyll and carotenoids were measured as described by Forth and Pyke [33]. Briefly, total chlorophyll from $2 \mathrm{~g}$ of fresh expanded leaves and total carotenoids from $3 \mathrm{~g}$ of fresh ripe fruits were extracted using hexane/acetone $(3: 2, v / v)$ and acetone/ petroleum ether $(1: 4, \mathrm{v} / \mathrm{v})$, respectively. After centrifugation, the supernatant was measured using spectrophotometer (PerkinElmer, USA). The amount was calculated with the following equations: total chlorophyll $\mathrm{mg} \mathrm{mL}^{-1}=8.02\left(\mathrm{OD}_{643}\right)+20.2\left(\mathrm{OD}_{647}\right)$, and total carotenoids $\mathrm{mg} \mathrm{mL}^{-1}=\left(\mathrm{OD}_{450}\right) / 0.25$.

For quantification of lycopene, $\beta$-carotene and lutein, pigments were extracted from $2 \mathrm{~g}$ of fresh ripe fruits using acetone/petroleum ether $(1: 1, v / \mathrm{v})$, then dried under a stream of $\mathrm{N}_{2}$ and dissolved in $100 \%$ dichloromethane. The HPLC analysis was performed with $10 \mu \mathrm{L}$ dichloromethane-dissolved pigments on ACQUITY UPLC (Waters, USA). Lycopene, $\beta$-carotene and lutein were identified by their characteristic absorption spectra (472 nm, $450 \mathrm{~nm}$ and $446 \mathrm{~nm}$, respectively) and distinctive retention times, compared with their corresponding standard substance (Sigma, USA). Each carotenoid content was calculated through the linear regression equation generated from the corresponding calibration curve, which was made using the corresponding standard substance. Individual tissue samples above were taken from 3 to 5 leaves or fruits for each line in triplicate.

\section{ABA measurement}

ABA was extracted from $100 \mathrm{mg}$ of pericarp tissues of fresh ripe fruits $(\mathrm{Br}+7$ days) using $1 \mathrm{~mL}$ of solution $\mathrm{I}$ ( $80 \%$ methanol, $19.95 \% \mathrm{H}_{2} \mathrm{O}$ and $0.05 \%$ acetic acid, $v / \mathrm{v}$ ). The supernatant was collected, dried under a stream of $\mathrm{N}_{2}$ and dissolved in $0.5 \mathrm{~mL}$ petroleum ether to remove the pigments. Then the subnatant was collected, dried under a stream of $\mathrm{N}_{2}$ and dissolved in $0.5 \mathrm{~mL}$ solution II (40\% methanol, 59.94\% $\mathrm{H}_{2} \mathrm{O}$ and $0.06 \%$ acetic acid, v/v). The HPLC analysis was carried out with $10 \mu \mathrm{L}$ solution II-dissolved ABA using ultraviolet/visible detector on ACQUITY UPLC (Waters, USA). Spectra were collected at $254 \mathrm{~nm}$, and $\mathrm{ABA}$ contents were calculated through the linear regression equation generated from the calibration curve, which was made using the standard substance of ABA (Sigma, USA). Individual tissue samples were taken from 3 to 5 fruits for each line in triplicate.

\section{Results}

SIIPT4 expression is predominant in ovary, sustained enhancement during fruit ripening, and regulated by drought stress

Knowing the expression patterns of a gene sometimes is helpful for knowledge about its physiological function, thus the levels of SIIPT4 expression in tomato were 
comprehensively examined using qRT-PCR. In about 8-week-old tomato plants, SIIPT4 mRNA was detectable in all organs, and shown strong abundance in roots, stems and leaves, moderate accumulation in flowers and weak level in fruits (Fig. 1a). Given the confirmed roles of CKs in the process of leaf senescence and at the early stages of fruit development, SIIPT4 transcripts were examined throughout leaf and fruit ontogeny. The mRNA accumulation of SIIPT4 is high in young leaves, and distinctly and continuously downregulated along with the process of leaf development and maturation (Fig. 1b). In flower, SIIPT4 expression is predominant in ovary, where SIIPT4 mRNA keeps enormous accumulation at bud ($2 \mathrm{dpa}$ ) and anthesis (0 dpa) stages, and displays dramatic downregulation from anthesis to post-anthesis (4 dpa) transition when fruit set is expected to occur (Fig. 1c). Subsequently, SIIPT4 expression maintains a moderate level at the mature green stage of fruit development. Interestingly, SIIPT4 expression displays a sustained enhancement along with fruit ripening, especially, obvious sharp upregulation from orange stage to red stage when lycopene biosynthesis is high active (Fig. 1d), which provides an important clue about its potential roles in the process of tomato fruit ripening.

The expression of SIIPT4 was significantly decreased by $40 \%$ under drought stress compared to the control plants (Fig. 1e). Interestingly, SIIPT4 mRNA didn't return toward the normal level at once after recovering watering, on the contrary, it decreased more seriously. After $6 \mathrm{~h}$ of restoration, the SIIPT4 transcripts increased gradually and reached 1.6 fold of that in the control plants at $24 \mathrm{~h}$. The results suggest that SIIPT4 may be involved in plant response to drought stress, where the phytohormone $\mathrm{ABA}$ is the best known trigger of drought tolerance in plants [34].

\section{SIIPT4 knockdown in tomato accelerated leaf senescence}

To characterize the physiological function of SIIPT4 in tomato, a loss-of-function approach was implemented using RNAi strategy. A total of more than ten transgenic lines were generated via Agrobacterium tumefaciens-mediated transformation. The most readily visible phenotype was related to leaf senescence. The wild-type tomato leaves were still green and alive at later growth stage of about 8-week-old plants (Fig. 2a). By contrast, the leaves of SIIPT4-RNAi transgenic lines turned into yellow color at expanded mature stage and displayed an accelerated senescence phenotype (Fig. 2b), and most of leaves withered and abscised from plants at 8-week-old stage (Fig. 2a). The level of SIIPT4 transcript was significantly reduced by more than $70 \%$ in transgenic lines compared with that in wild-type control plants (Fig. 2c), further supporting that downregulation of SIIPT4 accounted for the phenotypes displayed in the transgenic lines. The
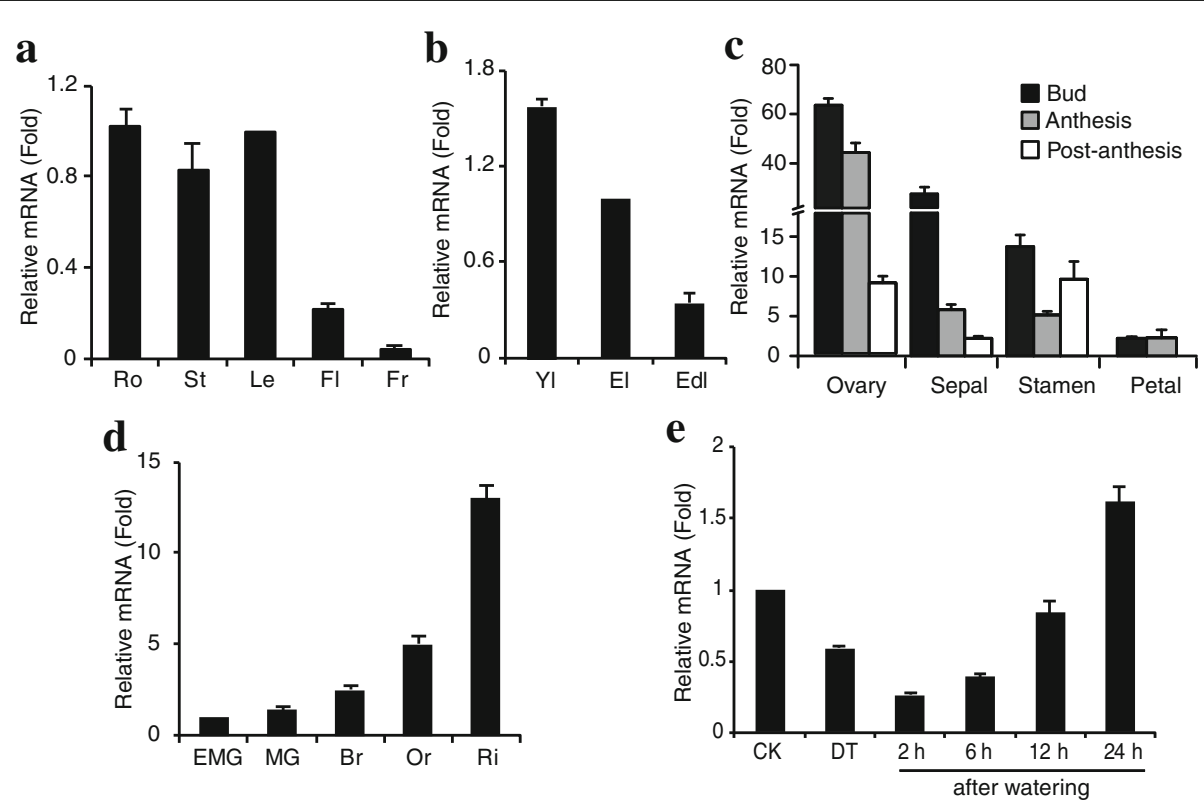

Fig. 1 Expression patterns of SIIPT4 in tomato. Relative expression analysis of SIIPT4 was performed in different tissues (a), in leaves at different developmental stages (b), in different parts of flower at different developmental stages (c), in fruits at different stages (d), and in response to drought stress treatment (e) by qRT-PCR. Petals have been shed at the post-anthesis stage, so no data was shown at this stage. Data are expressed as relative values, based on the values of leaf (a), expending leaf (b), early mature green fruit (c and $\mathbf{d}$ ), and corresponding control groups (e) taken as reference sample set to 1. Each value represents mean \pm SE (standard error) of three replicates. Ro, root; St, stem; Le, leaf; Fl, flower; Fr, fruit. Yl, young leaf; El, expending leaf; Edl, expended leaf. EMG, early mature green; MG, mature green; Br, break; Or, orange; Ri, ripening. CK, control; DT, drought treatment; $2,6,12$ and $24 \mathrm{~h}$ represent the hours after watering 
a

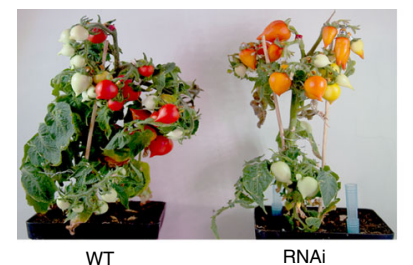

d

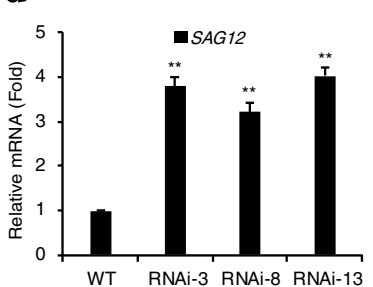

g

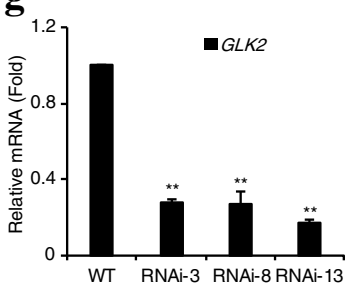

b

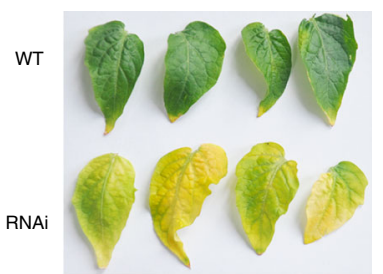

e

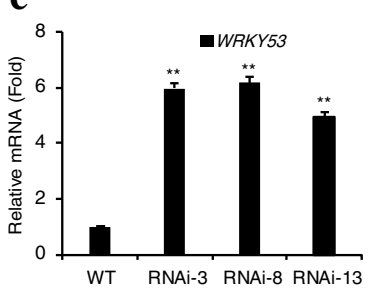

h

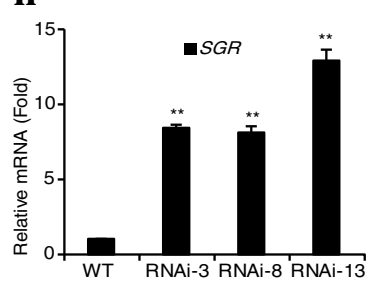

c

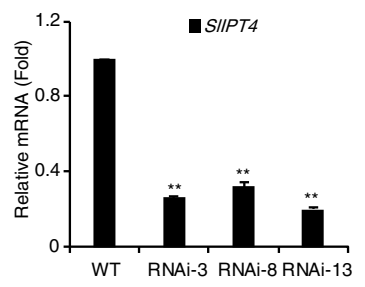

f

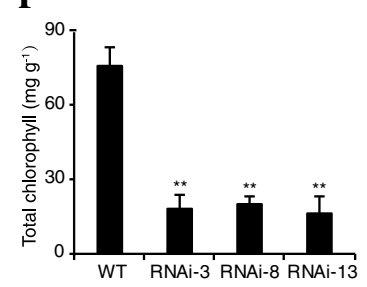

i

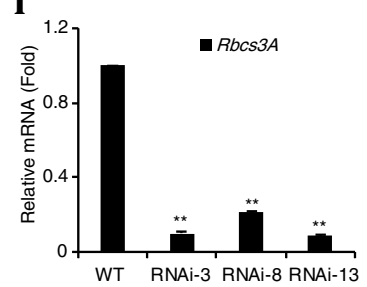

Fig. 2 SIIPT4 knockdown in tomato induced leaf senescence. $\mathbf{a}$ and $\mathbf{b}$ The phenotype of accelerated leaf senescence in SIIPT4-RNAi lines. Most leaves withered and abscised at 8-week-old plants (a), and leaves at expanded mature stage became yellow color (b). c SIIPT4 silencing efficiency was confirmed by qRT-PCR. $\mathbf{d}$ and e Relative expression levels of senescence marker genes, SAG12 and WRKY53, were tested by qRT-PCR. $\mathbf{f}$ Obvious reduction of total chlorophyll in expanded leaves of SIIPT4-RNAi lines as shown in $\mathbf{b}$. Biological replicates were performed in triplicate, and the data are shown as mean \pm SE. $\mathbf{g}$ and $\mathbf{h}$ and $\mathbf{i}$ Relative expression levels of GLK2, SGR, and Rbcs3A (regulating chlorophyll biosynthesis, chlorophyll degradation, and photosynthetic rate, respectively) were tested by qRT-PCR. For qRT-PCR, data are expressed as relative values based on the values of the control wild-type (WT) taken as reference sample set to 1. Each value represents mean \pm SE of three replicates. Asterisks represent significant differences between SIIPT4-RNAi line and wild-type control $(* P<0.05, * * P<0.01$, Student's $t$ test)

senescence specificity of senescence-associated gene $S A G 12$, encoding a cysteine protease, makes this gene as a molecular marker to study the senescence process [35]. The transcription factor WRKY53 regulates senescence specific gene expression [36]. Further the remarkable enhanced expression of SAG12 (GenBank no. XM_004233006) and WRKY53 (GenBank no. XM_004244582), indicated the accelerated senescence occurred in SIIPT4-RNAi leaves (Fig. 2d and e).

Knowing chlorophyll is predominant pigments in green leaves, plays an essential role in photosynthesis, and is degraded during the process of leaf senescence, it prompted us to measure chlorophyll content in leaves. At the expanded mature stage as shown in Fig. 2b, SIIPT4 silencing resulted in an about $75 \%$ reduction of total chlorophyll content in yellow leaves of SIIPT4-RNAi lines compared with that in green leaves of wild-type control plants (Fig. 2f). Considering chlorophyll homeostasis is mainly maintained through the dynamic balance between biosynthesis and degradation in plants, the expression levels of two crucial genes regulating these processes were determined by qRT-PCR. The transcription factor Golden2-like (GLK2) regulates positively chlorophyll biosynthesis through affecting chloroplast development [37]. STAY-GREEN (SGR) plays a decisive impact on chlorophyll degradation in plants [38]. Agreeing with the accelerated-senescence phenotype and the reduced chlorophyll accumulation in SIIPT4-RNAi leaves, the transcripts of GLK2 were remarkably decreased (Fig. 2g), while SGR expression was significantly upregulated (Fig. 2h), suggesting that SIIPT4 silencing could disturb chlorophyll accumulation through blocking chlorophyll biosynthesis and promoting the degradation of chlorophyll in leaves. The decrease of photosynthetic rate is also an important characteristic of leaf senescence. The dramatic downregulated expression of a key gene, ribulose-1,5-bisphosphate carboxylase small subunit (Rbcs3A) (Fig. 2i), in regulating photosynthetic rate [39], indicated a reduced photosynthetic rate in SIIPT4-RNAi leaves. It is well known that CKs can inhibit leaf senescence [40], however, its exact regulation mechanism is still unknown. Here phenotype and molecular analyses of SIIPT4-RNAi 
transgenic tomatoes support the hypothesis that SIIPT4 is involved in this regulatory network of leaf senescence.

\section{SIIPT4 silencing in tomato caused orange fruits and decreased lycopene accumulation}

Although the role of CKs in regulating cell division at the early stages of fruit development is well known, and SIIPT4 gene exhibits very strong expression level in ovaries and obvious dynamic expression in flowers during bud-to-anthesis and anthesis-to-post-anthesis transitions (Fig. 1c), the behaviors of fruit set and developmental process in all SIIPT4-silenced lines were indistinguishable from the control plants. However, surprisingly, the fruits of SIIPT4-RNAi lines couldn't normally ripen and displayed orange surface at about 7 days after breaker stage, while the fruits of wild-type control plants could become typical deep-red at the same growth stage (Fig. 3a). To reveal whether SIIPT4 downregulation delayed the progress of tomato fruit ripening, the orange fruits were held on the plants. However, the orange fruits of SIIPT4-RNAi lines didn't switch to red color even when plants died (see 0 day fruits in Fig. 3b). Moreover, these orange fruits also couldn't change the color after a long-time storage of about 30 days at room temperature (Fig. 3b). These results stated clearly that SIIPT4 is involved in regulating color formation during tomato fruit ripening, and its downregulation caused never red fruits.

Given the dramatic increase of carotenoids, especially lycopene during fruit ripening in tomato, the related carotenoids were measured in both SIIPT4-RNAi and wild-type fruits. Compared with wild-type control fruits, the contents of all carotenoids measured were reduced in SIIPT4-RNAi fruits (Fig. 3c-f). Total carotenoid levels were reduced by about $35 \%$ when quantified using spectrophotometric methods (Fig. 3c). However, HPLC-mediated quantification of lycopene accumulation showed a decrease of more than 75\% (Fig. 3d, Additional file 2: Table S2). Due lycopene is the predominant pigment and endows the ripe fruit with deep-red color, we concluded that the enormous reduction of lycopene accounted for the orange color in transgenic fruits. Meanwhile two derivatives of lycopene, $\beta$-carotene and lutein, were measured to assess the activity of lycopene catabolism. Similar to lycopene in SIIPT4-RNAi fruits, the contents of both $\beta$-carotene and lutein were also significantly reduced (Fig. 3e-f, Additional file 2: Table S2), suggesting the low content of lycopene in SIIPT4-RNAi fruits was not because of its biological catabolism. Therefore, repression of SIIPT4 expression leaded to the orange fruit phenotype through inhibiting lycopene biosynthesis.

\section{Expression of carotenoid biosynthetic genes were altered in SIIPT4-silenced fruits}

The carotenoid biosynthesis is mainly regulated at gene transcriptional level during fruit ripening [41]. Accordingly, to detect the mRNA levels of genes involved in lycopene biosynthesis and metabolism pathway is helpful to uncover the molecular regulation

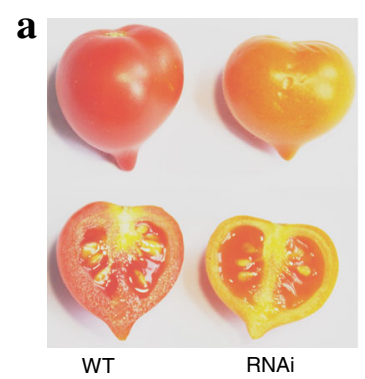

d

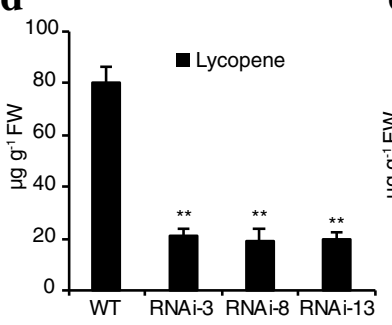

b

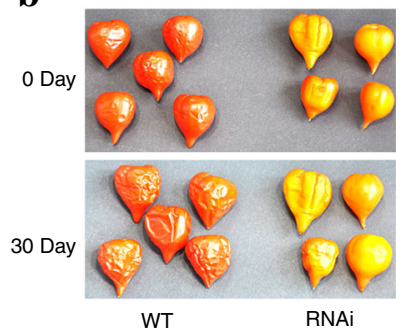

e

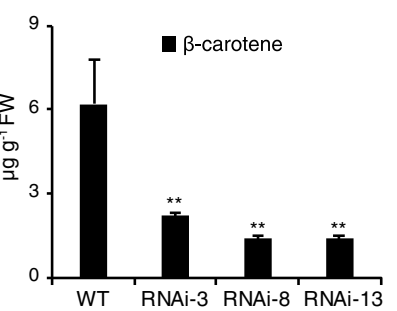

c

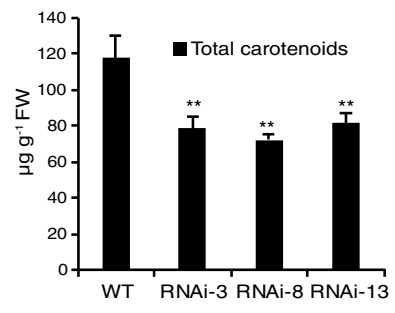

f

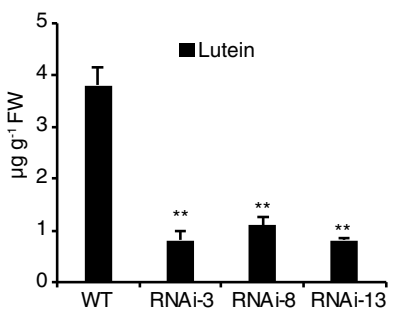

Fig. 3 SIIPT4 downregulation in tomato resulted in orange fruits and decreased lycopene accumulation. $\mathbf{a}$ and $\mathbf{b}$ Phenotypes of fruits at about 7 days after break stage (a), and after holding on plants until plant died (0 day) and a storage of 30 days at room temperature (b). $\mathbf{c}$, $\mathbf{d}$, e and $\mathbf{f} \mathrm{A}$ moderate reduction of total carotenoids (c) measured using spectrophotometer, and dramatic decrease of lycopene (d), $\beta$-carotene (e), and lutein (f) quantified via HPLC in SIIPT4-RNAi orange fruits. FW = fresh weight. The biological replicates (3-5 fruits per sample) were performed in triplicate, and the data are shown as mean \pm SE. Asterisks represent significant differences between SIIPT4-RNAi line and wild-type control $(* P<0.05, * * P<0.01$, Student's t test) 
mechanism of orange color fruits caused by SIIPT4 RNAi. As description above, the steps in lycopene biosynthesis pathway are orderly catalyzed by several crucial enzymes encoded by PSY1, PDS, ZISO, ZDS, and CrtISO genes, respectively (the pathway was shown in Fig. 6). Here the transcript levels of these genes were tested in 5-day fruits after break stage by qRT-PCR (Fig. 4). The first two genes, PSY1 and PDS, being responsible for colorless phytoene synthesis and desaturation to generate 9,15,9'-cis- $\zeta$-carotene, were markedly upregulated in SIIPT4-RNAi fruits. The ZISO gene required for the isomerization of $9,15,9^{\prime}$-cis- $\zeta$-carotene to $9,9^{\prime}$-cis- $\zeta$-carotene, was significantly downregulated by more than $80 \%$ in SIIPT4-RNAi fruits. The mRNA of ZDS, encoding a desaturase that catalyzes $9,9^{\prime}$-cis- $\zeta$-carotene to produce prolycopene, was mildly reduced in SIIPT4-RNAi lines. Moreover, SIIPT4 overexpression under the control of constitutive CaMV35S promoter increased the transcript accumulation of both ZISO and ZDS, and leaded to a little deep-redder fruits than wild-type control tomatoes (data not shown). The CrtISO and two CrtISO-like genes (CrtISO-L1 and CrtISO-L2) being responsible for the step of prolycopene to all-trans-lycopene in tomato, were upregulated by different degrees in SIIPT4-RNAi lines. In conclusion, we thought that the upregulated expression of PSY1,
PDS, and CrtISO genes was not the reasons for causing orange fruits in SIIPT4-RNAi lines, while the downregulation of ZISO and ZDS, especially the dramatic decrease of ZISO mRNAs, blocked the carotenoid biosynthesis pathway and resulted in the decreased accumulation of lycopene.

In the process of lycopene metabolism, two key cyclases, lycopene $\beta$-cyclase (LCYB) and lycopene $\varepsilon$-cyclase (LCYE), catalyze the formation of $\beta$-ring and $\varepsilon$-ring, respectively $[13,14]$. Although two products of lycopene metabolism, $\beta$-carotene and lutein, were reduced as described above (Fig. 3e-f), the expression levels of $L C Y B$ and $L C Y E$ were moderately increased in SIIPT4-RNAi lines (Fig. 4). This paradox might due the enhanced catabolism of $\beta$-carotene and lutein, or that low lycopene accumulation caused the reduction of lycopene metabolism products, which regulated the expression of their related catalyzing enzyme genes through a negative feedback mechanism.

\section{SIIPT4 silencing caused a reduction of ABA content and upregulated expression levels of $A B A$ biosynthesis-related genes}

Considering that the biosynthesis of ABA from the oxidative cleavage of $\beta$-carotene is the main pathway in higher plants, and $A B A$ feedback-regulates the
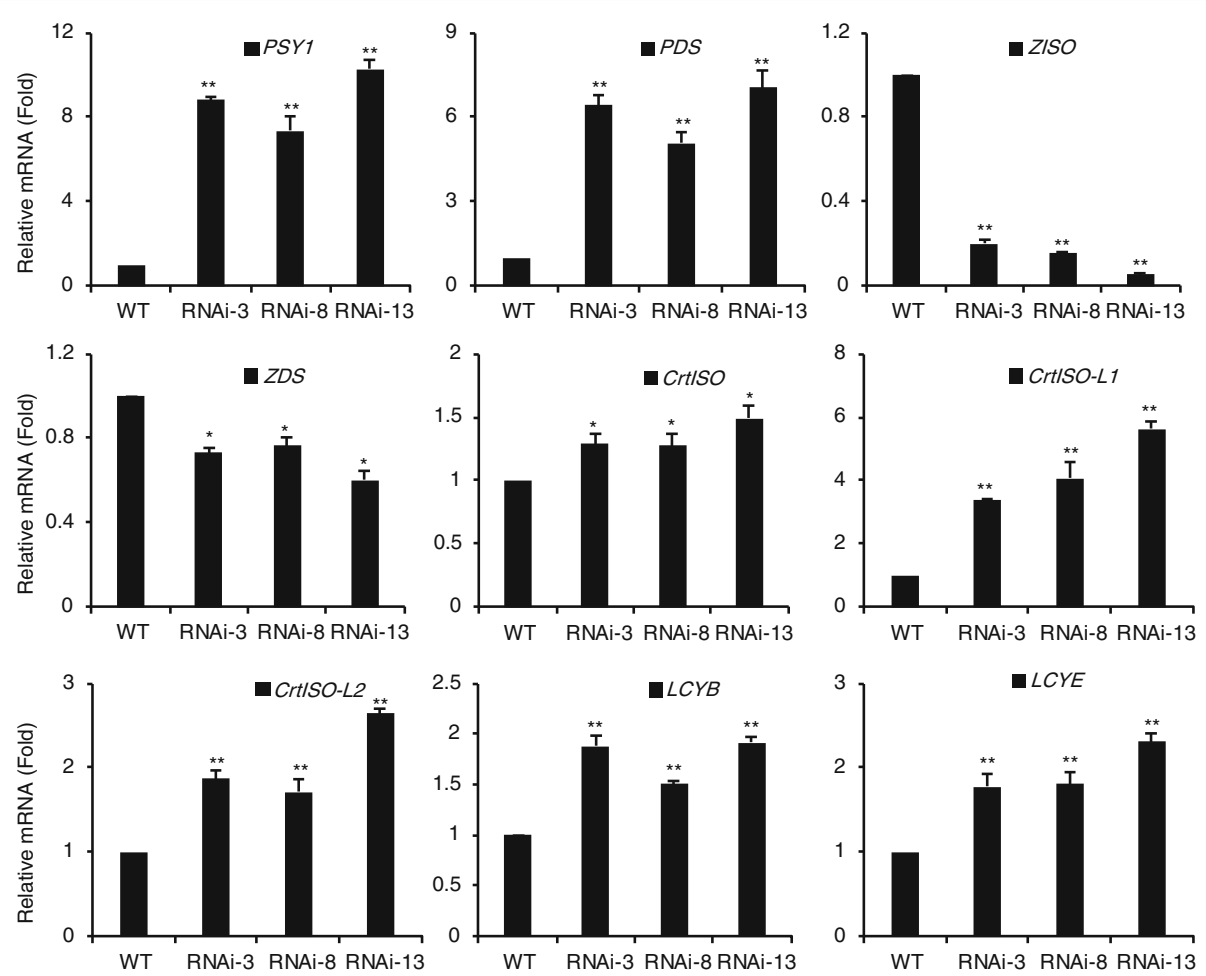

Fig. 4 Altered expression of genes in lycopene biosynthesis and metabolism pathway in SIIPT4-RNAi orange fruits. The relative level of transcripts were detected via qRT-PCR between wild-type and SIIPT4 RNAi lines. The data of wild-type (WT) were taken as reference and normalized to 1. Each value represents mean \pm SE of three replicates. Asterisks represent significant differences between SIIPT4-RNAi line and wild-type control $(* P$ $<0.05, * * P<0.01$, Student's t test) 
carotenoid biosynthesis $[3,42]$, the ABA content and the expression levels of ABA biosynthesis-related genes were tested to assess whether the decrease of $\beta$-carotene content affected ABA biosynthesis in SIIPT4-RNAi lines. The obvious reduction of ABA content in SIIPT4-RNAi fruits (Fig. 5a), proved that the reason of the low content of lycopene metabolism products is not their catabolism to produce $A B A$, but their weak biosynthetic activity caused by the very low accumulation of lycopene. In ABA biosynthesis pathway, zeaxanthin epoxidase (ZEP) and 9-cis-epoxycarotenoid dioxygenase (NCED) play key roles in higher plants. The ZEP catalyzes the formation of violaxanthin from zeaxanthin [43], and NCED controls the first committed and rate-limiting step of ABA biosynthesis from carotenoid pathway [44]. The upregulation expression of both NEP and NCED (NCED1 and NCED2) in orange fruits of transgenic lines (Fig. 5b-d), suggested that ABA biosynthesis genes were negative feedback regulated by the level of ABA accumulation.

\section{Discussion}

Leaf senescence is an internally programed phase with the redistribution of micro- and macro-nutrients from leaves to reproductive organs [40, 45-47]. Premature senescence in leaves generally results in reduced crop production and poor grain quality, while delaying leaf senescence can prolong the photosynthesis time and provide sufficient assimilated carbon to grain during the grain-filling period, thereby increasing crop yield. In plants, it has been confirmed that phytohormones are crucial in the regulation of leaf senescence, and among the various plant hormones, CKs have received the maximum attention for their roles in depressing leaf senescence [40]. For instance, exogenous application of CKs has the ability to delay the senescence of detached leaf in dark condition [48]. Although CKs play key roles in regulating leaf senescence, little is known about their exact regulation mechanism. Furthermore, endogenous CKs include several different forms, such as trans-zeatin (tZ), isopentenyladenosine (iPR), dihydrozeatin riboside (DZR), and isopentenyladenine (iP), each of them may play different roles in plants. It is puzzled which one or several CKs control the process of leaf senescence. The isopentenyltransferases (IPTs) are the rate-limiting enzymes catalyzing the initial step of CK biosynthesis, and are thought to have a different role in producing different CK among them [49]. Thus it is very necessary to illuminate the functions of IPTs. In the present study, we demonstrated that repressing expression of SIIPT4 accelerated leaf senescence in tomato, which is active and useful to discover the molecular mechanism of CKs regulating leaf senescence.

The prominent visible color change (from green to yellow) of leaf senescence is mainly caused by the degradation of green pigment chlorophyll [50]. Chlorophyll catabolism is a multistep pathway regulated by many

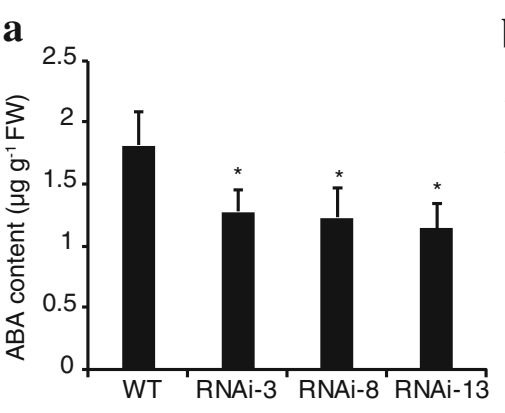

b
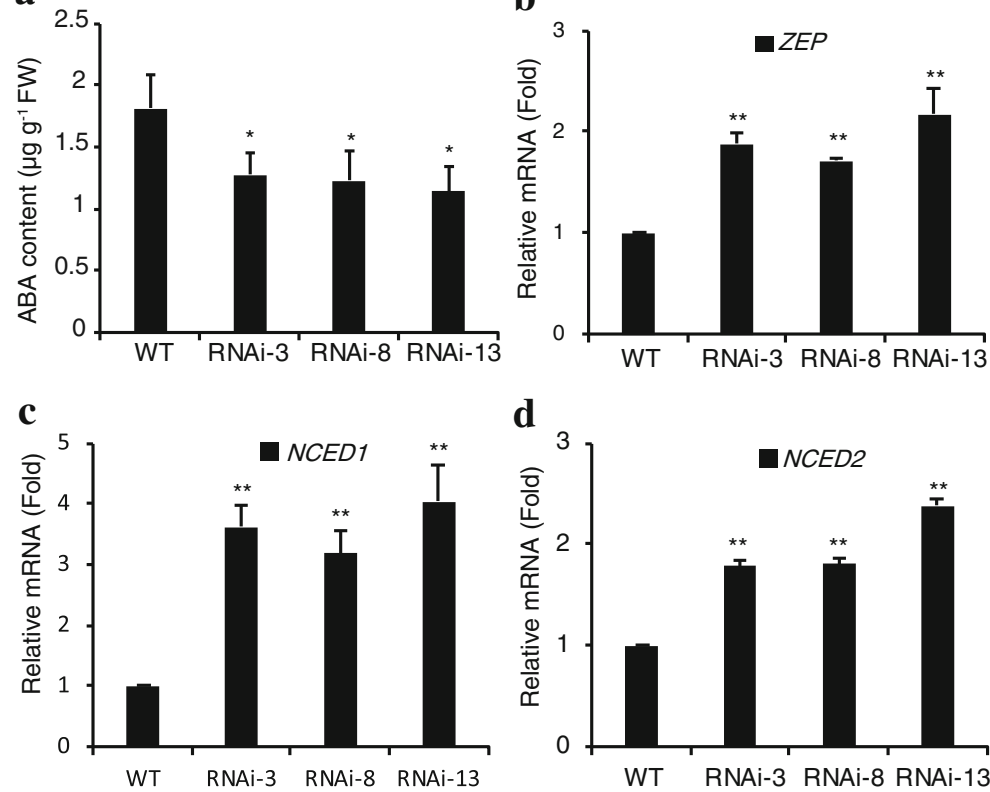

d

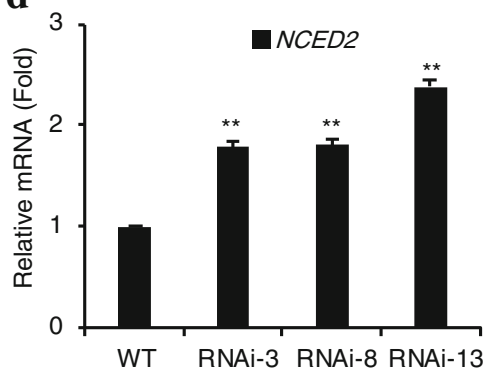

Fig. 5 Reduced accumulation of ABA in SIIPT4-RNAi orange fruits. a ABA content was measured using HPLC analysis. b, $\mathbf{c}$ and $\mathbf{d}$ Relative expression levels of ABA biosynthesis-related genes were tested by qRT-PCR. The data of wild-type (WT) were taken as reference and normalized to 1. Each value shown as mean \pm SE of three replicates. Asterisks represent significant differences between SIIPT4-RNAi line and wild-type control $(* P<0.05, * * P<0.01$, Student's t test) 
factors [40]. And among them, the SGR protein, characterized from stay green mutant, plays crucial roles in the regulation of chlorophyll degradation through dismantling photosynthetic chlorophyll-protein complexes and thus allowing chlorophyll-breakdown enzymes to access their substrate [51-55]. Repression of $S G R$ expression delayed chlorophyll degradation in tomato fruits and leaves, while constitutive expression of SGR accelerated chlorophyll breakdown in leaves [54]. In SIIPT4-silenced leaves, the level of $S G R$ mRNA was remarkably increased by about $8-12$ fold (Fig. 2 h), consistent with the decreased content of total chlorophyll (Fig. 2f). Besides enhanced degradation of chlorophyll, the chlorophyll biosynthesis-related gene, GLK2, was decreased in SIIPT4-silenced leaves (Fig. 2g). Moreover, the decline in photosynthetic capability is also the vital feature of leaf senescence, and the expression of Rbcs $3 A$, encoding a positive regulator of photosynthesis, was significantly downregulated (Fig. 3i), suggesting the reduced photosynthesis efficiency in SIIPT4-RNAi lines. Taken together, these data indicated that SIIPT4 is assuredly necessary for controlling leaf senescence.

It was expected that repression of SIIPT4 expression accelerated leaf senescence. However, it was out of our expectation that SIIPT4 knockdown did not cause any obvious phenotypic changes during fruit developmental stages, especially the cell division stage. Although the roles of CKs in fruit development are well known, not all types of CKs have effects on this process. For example, after applications of exogenous CKs in unpollinated ovaries of tomato, tZ, 6-benzylaminopurine (BA), and kinetin didn't have any visible effect on fruit set and growth, it was similar to untreated control ovaries with phenotype of neither abscission nor growth, however, application of $\mathrm{N}$-(2-chloro-pyridin-4yl)- $\mathrm{N}^{\prime}$-phenylurea (CPPU) induced growth [27]. Thus, we think no visible effect on fruit development in SIIPT4-RNAi lines may be due that there is potential functional redundancy among $I P T$ genes during fruit development, or this kind of CK produced by SIIPT4 may not be pivotal for this process.

CKs are thought to be important for fruit development, especially cell division [27], to our knowledge, their roles in fruit ripening have no reports so far. Interestingly, the mRNA level of SIIPT4 gradually increases during fruit ripening in tomato (Fig. 1d). More surprisingly, repressing the expression of SIIPT4 resulted in producing orange fruits, which were never red (Fig. 3a-b). As the predominant pigment in ripe tomato fruits, lycopene content was tremendously decreased (Fig. 3d, Additional file 2: Table S2), suggesting SIIPT4 silencing dramatically effected lycopene biosynthesis. During fruit ripening, chloroplasts convert to chromoplasts, which are pigment-filled plastids responsible for the bright colors. Thus the subcellular localization of SIIPT4 in the chloroplast and the cytosolic area surrounding chloroplasts uncovered by Žižková et al. [31], strongly supports our results that SIIPT4 is involved in regulating leaf senescence and fruit color formation.

Among lycopene biosynthesis-related genes, SIIPT4 RNAi resulted in remarkable decrease of ZISO mRNA, moderate downregulation of $Z D S$ transcript, and obvious upregulated expression of PSY1, PDS, and CrtISOs (Fig. 4). ZISO is first defined by the maize $\mathrm{y} 9$ and present in single copy in tomato, Arabidopsis and grape [10, 56, 57]. In tomato, ZISO widely expresses in flower, root, leaf and fruit, and maintains high expression level during fruit ripening [10]. Silencing the expression of ZISO via virus-induced gene silencing (VIGS) in tomato resulted in pale-red fruits with a distinct reduction of lycopene and a compensatory increased accumulation of phytoene, phytofluene, and $\zeta$-carotene [10]. Similarly, the recessive mutant zeta $\left(z^{2803}\right)$, a mutation in ZISO gene, blocks carotenoid biosynthesis in fruits at $\zeta$-carotene with almost undetectable lycopene [58]. Moreover, SIIPT4 overexpression could enhance the mRNA levels of both $Z I S O$ and $Z D S$, and give rise to a little deep-redder fruits (data not shown). Taken together, these data strongly supported that downregulation of SIIPT4 expression in tomato fruit inhibited carotenoids biosynthesis, especially caused a sharp reduction of lycopene content, through repressing the expression of ZISO.

The puzzling complex regulatory loops regulating the abundance of key transcripts in response to the operation of the carotenoid biosynthesis pathway is existent in tomato, it was discussed previously by Kachanovsky et al. [58] and Fantini et al. [10]. The level of product feedback regulates the transcript induction of its upstream genes in carotenoid biosynthesis pathway. For example, the $P D S$ promoter is induced when carotenoid accumulation is repressed in tomato leaves [59]; Prolycopene or its metabolite was hypothesized as the signal mediating PSY1 induction [58]; Fantini et al. thought a minimum level of prolycopene is required to attain $P S Y 1$ transcript induction [10]. The transcript level of ZISO was highly correlated with mRNAs of $P D S$, encoding the enzyme that produces the substrate for ZISO [10]. The $Z I S O$ and CrtISO were induced in PDS- and ZDS-silenced fruits, respectively [10]. The downregulation of $Z I S O$ and $Z D S$, and the upregulation of other genes (PSY1, PDS, CrtISO, CrtISO-L1, and CrtISO-L2) involved in carotenoid biosynthesis in SIIPT4-silenced fruits with very low content of lycopene (Figs. 3 and 4), supports the hypothesis of the existence of regulatory loops in the carotenoid biosynthesis pathway.

It has been confirmed that $\mathrm{ABA}$ is mainly derived from carotenoids pathway in higher plants, and low content of ABA caused by disruption of carotenoid biosynthesis or active biosynthesis of $\mathrm{ABA}$ induces $P S Y$ transcription 
through feedback regulation mechanism (reviewed in ref. $[3,58])$. For example, under abiotic stress of saline or drought, the increased demand for ABA to trigger stress tolerance drives PSY expression to enhance carotenoid biosynthesis pathway for providing more xanthophylls of ABA precursors in roots [60, 61]. In SIIPT4-RNAi fruits, the ABA accumulation was reduced due to the repression of carotenoid biosynthesis pathway (Fig. 5a), while the upregulated expression of $\mathrm{ABA}$ biosynthesis-related genes (ZEP, NCED1 and NECD2) (Fig. 5b-d) indicated that the transgenic plants might try to restore the normal level of ABA. Accordingly, the low accumulation of ABA in SIIPT4-RNAi fruits was likely to feedback regulate carotenoid biosynthesis pathway for trying to promote the supply of ABA precursors through inducing PSY1 expression, which accords with this feedback regulation system between ABA content and PSY1 expression.

The decreased content of CKs in ipt1 357 mutants or overexpression transgenic plants of $C K X$, encoding a cytokinin oxidase/dehydrogenase catalyzing the irreversible degradation of CKs, could significantly reduce ABA accumulation in Arabidopsis thaliana [62]. SlIPT4 expression is very sensitive to drought stress (Fig. 1e), where $\mathrm{ABA}$ is the best known trigger of drought tolerance in plants [34]. These results suggest that SlIPT4 may be associated with ABA biosynthesis. Accordingly, both the potential reduction of CK content caused by
SIIPT4 silencing and the sharp repression of carotenoid biosynthesis might result in a reduced accumulation of ABA in SIIPT4-RNAi fruits.

Consequently, based on the results and analysis above, we raised a proposed regulation model for the roles of SIIPT4 in the control of lycopene biosynthesis and leaf senescence in tomato (Fig. 6). In this model, SIIPT4 gene regulates the lycopene biosynthesis via controlling the expression level of ZISO. The ABA accumulation is regulated by SIIPT4 through two potential ways: one way is that the alteration of carotenoid biosynthesis affects directly the supply of ABA precursors; and another way is that ABA accumulation is regulated by the potential correlation between CKs and ABA. Then the alteration of ABA content feedback regulates the expression level of the first gene PSY1 in carotenoid biosynthesis pathway. Though ABA was discovered to be involved in leaf senescence, however, due to the known role of CKs in leaf senescence, we thought that the SIIPT4-mediated leaf senescence might be mainly attributed to affect the corresponding CK biosynthesis in tomato. Nevertheless, the exact mechanism through which the effects of SIIPT4 are exerted in tomato leaves and fruits needs and deserves further investigation.

\section{Conclusion}

Most studies devoted so far to IPT genes focused on their roles in the known cytokinin-related process, such

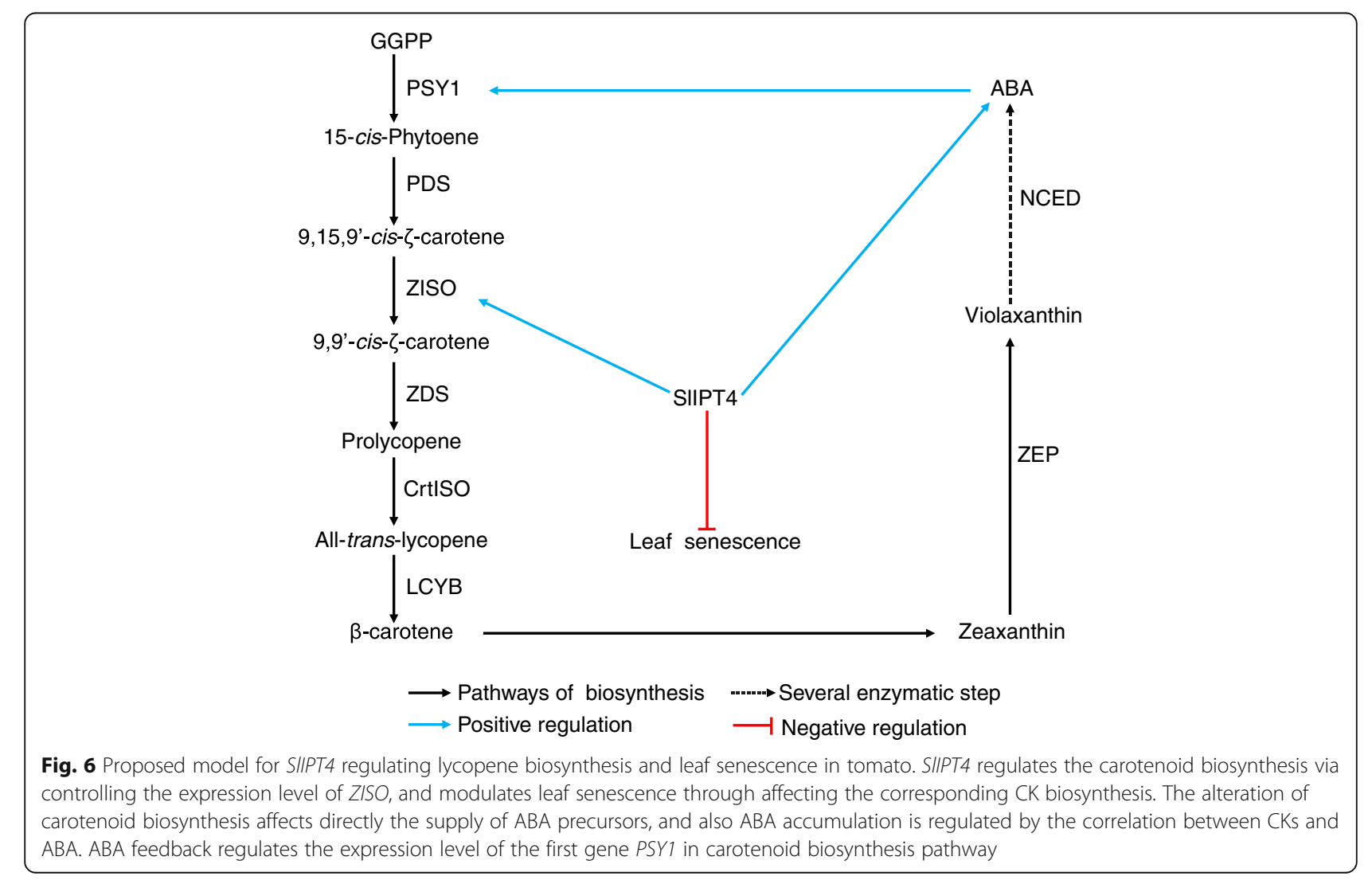


as cell division, apical dominance, leaf senescence and fruit development, as well as in response to biotic and abiotic stress. Here, we uncovered the functional roles of SIIPT4 in not only leaf senescence but also the regulation of lycopene biosynthesis in tomato. The downregulation of SIIPT4 repressed carotenoids biosynthesis and reduced dramatically the accumulation of lycopene in tomato fruits. Among lycopene biosynthesis-related genes, dramatic reduction of ZISO transcripts, moderate decrease of ZDS mRNAs, and upregulation of other genes (PSY1, PDS, CrtISOs), suggested that SIIPT4 controls lycopene biosynthesis likely through affecting ZISO expression, and also supported the existence of regulatory loops in carotenoids/lycopene biosynthesis pathway. The decrease of ABA content and upregulation of PSY1 expression in SIIPT4-RNAi fruits, implied the feedback regulation between $\mathrm{ABA}$ and carotenoids biosynthesis. To our knowledge, this is the first report that cytokinin biosynthesis-related gene is involved in color formation during fruit ripening.

\section{Additional files}

Additional file 1: Table S1. Primers used for qRT-PCR. (PDF $37 \mathrm{~kb}$ )

Additional file 2: Table S2. Carotrnoids in fruits of wild-type and SIIPT4RNAi tomato plants. (PDF $61 \mathrm{~kb}$ )

\section{Abbreviations}

ABA: Abscisic acid; CKs: Cytokinins; CrtISO: Carotene isomerase; dpa: Day post anthesis; GLK2: Golden2-like; IPT: Isopentenyltransferases; LCYB: Lycopene- $\beta$ cyclase; LCYE: Lycopene $\varepsilon$-cyclase; NCED: 9-cis-epoxycarotenoid dioxygenase; PDS: Phytoene desaturase; PSY: Phytoene synthase; qRT-PCR: quantitative real-time PCR; Rbcs3A: Ribulose-1,5-bisphosphate carboxylase small subunit; RNAi: RNA interference; SGR: STAY-GREEN; ZDS: \-carotene desaturase; ZEP: Zeaxanthin epoxidase; ZISO: Z-carotene isomerase

\section{Funding}

This work was supported by the National Natural Science Foundation of China (31372080), the Committee of Science and Technology of Chongqing, China (cstc2017jcyjAX0455), and the Fundamental Research Funds for the Central Universities (106112013CDJZR290035).

\section{Availability of data and materials}

The datasets supporting the conclusions of this article are included within the article and its additional files. All plant materials were obtained from Chongqing University, Chongqing, China.

\section{Authors' contributions}

YY and LZ planned and designed the research. ZY, TY and CW performed the experiments. $Z Y$ and $Y W$ analyzed the data and wrote the manuscript. All authors read and approved the final manuscript.

\section{Ethics approval and consent to participate}

Not applicable.

\section{Competing interests}

The authors declare that they have no competing interests.

\section{Publisher's Note}

Springer Nature remains neutral with regard to jurisdictional claims in published maps and institutional affiliations.

\section{Author details}

${ }^{1}$ Bioengineering College, Chongqing University, Chongqing 400044, China.

${ }^{2}$ School of Life Sciences, Chongqing University, Chongqing 400044, China.

Received: 7 December 2017 Accepted: 24 May 2018

Published online: 05 June 2018

\section{References}

1. Britton G, Liaaen-Jensen S, Pfander H. Carotenoids Handbook. Basel: Birkhäuser Verlag; 2004.

2. Cazzonelli Cl, Pogson BJ. Source to sink: regulation of carotenoid biosynthesis in plants. Trends Plant Sci. 2010;15(5):266-74.

3. Ruiz-Sola MÁ, Rodríguez-Concepción M. Carotenoid biosynthesis in Arabidopsis: a colorful pathway. Arabidopsis Book. 2012;10:e0158.

4. Demming-Adams B, Gilmore AM, Adams WW. Carotenoids 3: in vivo functions of carotenoids in higher plants. FASEB J. 1996;10(4):403-12.

5. Xie X, Yoneyama K, Yoneyama K. The strigolactone story. Annu Rev Phytopathol. 2010;48:93-117.

6. Liu L, Shao Z, Zhang M, Wang Q. Regulation of carotenoid metabolism in tomato. Mol Plant. 2015;8(1):28-39.

7. DellaPenna D, Pogson BJ. Vitamin synthesis in plants: tocopherols and carotenoids. Annu Rev Plant Biol. 2006;57:711-38.

8. Krinsky NI, Johnson EJ. Carotenoid actions and their relation to health and disease. Mol Asp Med. 2005;26(6):459-516.

9. Rao AV, Agarwal S. Role of antioxidant lycopene in cancer and heart disease. J Am Coll Nutr. 2000;19(5):563-9.

10. Fantini E, Falcone G, Frusciante S, Giliberto L, Giuliano G. Dissection of tomato lycopene biosynthesis through virus-induced gene silencing. Plant Physiol. 2013;163(2):986-98.

11. Hirschberg J. Carotenoid biosynthesis in flowering plants. Curr Opin Plant Biol. 2001;4(3):210-8.

12. Fraser PD, Bramley PM. The biosynthesis and nutritional uses of carotenoids. Prog Lipid Res. 2004;43(3):228-65.

13. Pecker I, Gabbay R, Cunningham FX Jr, Hirschberg J. Cloning and characterization of the cDNA for lycopene beta-cyclase from tomato reveals decrease in its expression during fruit ripening. Plant Mol Biol. 1996;30(4):807-19.

14. Cunningham FX Jr, Gantt E. One ring or two? Determination of ring number in carotenoids by lycopene $\varepsilon$-cyclases. Proc Natl Acad Sci U S A. 2001;98(5):2905-10.

15. Giovannoni JJ. Genetic regulation of fruit development and ripening. Plant Cell. 2004;16(Suppl):S170-80.

16. Shi J, Le Maguer M. Lycopene in tomatoes: chemical and physical properties affected by food processing. Crit Rev Food Sci Nutr. 2000;40(1):1-42.

17. Ronen G, Cohen M, Zamir D, Hirschberg J. Regulation of carotenoid biosynthesis during tomato fruit development: expression of the gene for lycopene epsilon-cyclase is down-regulated during ripening and is elevated in the mutant Delta. Plant J. 1999;17(4):341-51.

18. Fray RG, Grierson D. Identification and genetic analysis of normal and mutant phytoene synthase genes of tomato by sequencing, complementation and co-suppression. Plant Mol Biol. 1993;22(4):589-602.

19. Isaacson $T$, Ronen $G$, Zamir D, Hirschberg J. Cloning of tangerine from tomato reveals a carotenoid isomerase essential for the production of $\beta$ carotene and xanthophylls in plants. Plant Cell. 2002;14(2):333-42.

20. Martel C, Vrebalov J, Tafelmeyer P, Giovannoni JJ. The tomato MADS-box transcription factor RIPENING INHIBITOR interacts with promoters involved in numerous ripening processes in a COLORLESS NONRIPENING-dependent manner. Plant Physiol. 2011;157(3):1568-79.

21. Luo Z, Zhang J, Li J, Yang C, Wang T, Ouyang B, et al. A STAY-GREEN protein SISGR1 regulates lycopene and $\beta$-carotene accumulation by interacting directly with SIPSY1 during ripening processes in tomato. New Phytol. 2013;198(2):442-52.

22. Chung MY, Vrebalov J, Alba R, Lee J, McQuinn R, Chung JD, et al. A tomato (Solanum lycopersicum) APETALA2/ERF gene, SIAP2a, is a negative regulator of fruit ripening. Plant J. 2010;64(6):936-47.

23. Welsch R, Maass D, Voegel T, DellaPenna D, Beyer AP. Transcription factor RAP2.2 and its interacting partner SINAT2: stable elements in the carotenogenesis of Arabidopsis leaves. Plant Physiol. 2007;145(3):1073-85.

24. Toledo-Ortiza G, Huqb E, Rodríguez-Concepción M. Direct regulation of phytoene synthase gene expression and carotenoid biosynthesis by phytochrome-interacting factors. Proc Natl Acad Sci U S A. 2010;107(25): 11626-31. 
25. Werner T, Motyka V, Laucou V, Smets R, Van Onckelen H, Schmülling T. Cytokinin-deficient transgenic Arabidopsis plants show multiple developmental alterations indicating opposite functions of cytokinins in the regulation of shoot and root meristem activity. Plant Cell 2003;15(11):2532-2550.

26. Argueso CT, Ferreira FJ, Kieber JJ. Environmental perception avenues: the interaction of cytokinin and environmental response pathways. Plant Cell Environ. 2009;32(9):1147-60.

27. Matsuo S, Kikuchi K, Fukuda M, Honda I, Imanishi S. Roles and regulation of cytokinins in tomato fruit development. J Exp Bot. 2012;63(15):5569-79.

28. Ha S, Vankova R, Yamaguchi-Shinozaki K, Shinozaki K, Tran LP. Cytokinins: metabolism and function in plant adaptation to environmental stresses. Trends Plant Sci. 2012;17(3):172-9.

29. Kakimoto T. Identification of plant cytokinin biosynthetic enzymes as dimethylallyl diphosphate:ATP/ADP isopentenyltransferases. Plant Cell Physiol. 2001;42(7):677-85.

30. Takei K, Sakakibara H, Sugiyama T. Identification of genes encoding adenylate isopentenyltransferase, a cytokinin biosynthesis enzyme, in Arabidopsis thaliana. J Biol Chem. 2001;276(28):26405-10.

31. Žižková E, Dobrev PI, Muhovski Y, Hošek P, Hoyerová K, Haise D, et al. Tomato (Solanum lycopersicum L.) SIIPT3 and SIIPT4 isopentenyltransferases mediate salt stress response in tomato. BMC Plant boil. 2015;15:85.

32. Yang Y, Wu Y, Pirrello J, Regad F, Bouzayen M, Deng W, Li Z. Silencing SIEBF1 and SI-EBF2 expression causes constitutive ethylene response phenotype, accelerated plant senescence, and fruit ripening in tomato. $J$ Exp Bot. 2010;61(3):697-708.

33. Forth D, Pyke KA. The suffulta mutation in tomato reveals a novel method of plastid replication during ripening. J Exp Bot. 2006;57(9):1971-9.

34. Okamoto M, Peterson FC, Defries A, Park SY, Endo A, Nambara E, et al. Activation of dimeric ABA receptors elicits guard cell closure, ABA-regulated gene expression, and drought tolerance. Proc Natl Acad Sci U S A. 2013; 110(29):12132-7.

35. Noh YS, Amasino RM. Identification of a promoter region responsible for the senescence-specific expression of SAG12. Plant Mol Biol. 1999;41(2):181-94.

36. Miao Y, Laun T, Zimmermann P, Zentgraf U. Targets of the WRKY53 transcription factor and its role during leaf senescence in Arabidopsis. Plant Mol Biol. 2004;55(6):853-67.

37. Waters MT, Moylan EC, Langdale JA. GLK transcription factors regulate chloroplast development in a cell-autonomous manner. Plant J. 2008;56(3): 432-44

38. Park SY, Yu JW, Park JS, Li J, Yoo SC, Lee SK, et al. The senescence-induced staygreen protein regulates chlorophyll degradation. Plant Cell. 2007;19(5): 1649-64.

39. Ueda T, Pichersky E, Malik VS, Cashmore AR. Level of expression of the tomato $r b c S-3 A$ gene is modulated by a far upstream promoter element in a developmentally regulated manner. Plant Cell. 1989;1(2):217-27.

40. Sarwat M, Naqvi AR, Ahmad P, Ashraf M, Akram NA. Phytohormones and microRNAs as sensors and regulators of leaf senescence: assigning macro roles to small molecules. Biotechnol Adv. 2013;31(8):1153-71.

41. Bramley PM. Regulation of carotenoid formation during tomato fruit ripening and development. J Exp Bot. 2002;53(377):2107-13.

42. Finkelstein R. Abscisic acid synthesis and response. Arabidopsis Book. 2013; 11:e0166.

43. Wang N, Fang W, Han H, Sui N, Li B, Meng QW. Overexpression of zeaxanthin epoxidase gene enhances the sensitivity of tomato PSII photoinhibition to high light and chilling stress. Physiol Plant. 2008;132(3): 384-96

44. Sun L, Yuan B, Zhang M, Wang L, Cui M, Wang Q, Leng P. Fruit-specific RNAi-mediated suppression of SINCED1 increases both lycopene and $\beta$ carotene contents in tomato fruit. J Exp Bot. 2012;63(8):3097-108.

45. Balazadeh S, Riañopachón DM, Mueller-Roeber B. Transcription factors regulating leaf senescence in Arabidopsis thaliana. Plant Biol. 2008;10(Suppl 1):63-75.

46. Jibran $R, A$ Hunter D, P Dijkwel P. Hormonal regulation of leaf senescence through integration of developmental and stress signals. Plant Mol Biol. 2013;82(6):547-61.

47. Zhang $H$, Zhou C. Signal transduction in leaf senescence. Plant Mol Biol. 2013;82(6):539-45.

48. Talla SK, Panigrahy M, Kappara S, Nirosha P, Neelamraju S, Ramanan R. Cytokinin delays dark-induced senescence in rice by maintaining the chlorophyll cycle and photosynthetic complexes. J Exp Bot. 2016;67(6): 1839-51.
49. Kieber JJ, Schalle GE. Cytokinins. Arabidopsis Book. 2014;12:e0168.

50. Hörtensteiner S. Chlorophyll degradation during senescence. Annu Rev Plant Biol. 2006;57:55-77.

51. Jiang H, Li M, Liang N, Yan H, Wei Y, Xu X, et al. Molecular cloning and function analysis of the stay green gene in rice. Plant J. 2007;52(2):197-209.

52. Ren G, An K, Liao Y, Zhou X, Cao Y, Zhao H, et al. Identification of a novel chloroplast protein atnye1 regulating chlorophyll degradation during leaf senescence in Arabidopsis. Plant Physiol. 2007;144(3):1429-41.

53. Hörtensteiner S. Stay-green regulates chlorophyll and chlorophyll-binding protein degradation during senescence. Trends Plant Sci. 2009;14(3):155-62.

54. Hu ZL, Deng L, Yan B, Pan Y, Luo M, Chen XQ, et al. Silencing of the LeSGR1 gene in tomato inhibits chlorophyll degradation and exhibits a stay-green phenotype. Biol Plantarum. 2011;55(1):27-34

55. Zhou C, Han L, Pislariu C, Nakashima J, Fu C, Jiang Q, et al. From model to crop: functional analysis of a STAY-GREEN gene in the model legume Medicago truncatula and effective use of the gene for alfalfa improvement. Plant Physiol. 2011;157(3):1483-96.

56. Chen Y, Li F, Wurtzel ET. Isolation and characterization of the Z-ISO gene encoding a missing component of carotenoid biosynthesis in plants. Plant Physiol. 2010;153(1):66-79.

57. Li F, Murillo C, Wurtzel ET. Maize y9 encodes a product essential for 15-cis- $\zeta$ carotene isomerization. Plant Physiol. 2007;144(2):1181-9.

58. Kachanovsky DE, Filler S, Isaacson T, Hirschberg J. Epistasis in tomato color mutations involves regulation of phytoene synthase 1 expression by ciscarotenoids. Proc Natl Acad Sci U S A. 2012;109(46):19021-6.

59. Corona V, Aracri B, Kosturkova G, Bartley GE, Pitto L, Giorgetti L, Scolnik PA, Giuliano G. Regulation of a carotenoid biosynthesis gene promoter during plant development. Plant J. 1996;9(4):505-12.

60. Arango J, Wüst F, Beyer $P$, Welsch $R$. Characterization of phytoene synthases from cassava and their involvement in abiotic stress-mediated responses. Planta. 2010;232(5):1251-62.

61. Welsch R, Wüst F, Bär C, Al-Babili S, Beyer P. A third phytoene synthase is devoted to abiotic stress-induced abscisic acid formation in rice and defines functional diversification of phytoene synthase genes. Plant Physiol. 2008; 147(1):367-80.

62. Nishiyama R, Watanabe Y, Fujita Y, Le DT, Kojima M, Werner T, et al. Analysis of cytokinin mutants and regulation of cytokinin metabolic genes reveals important regulatory roles of cytokinins in drought, salt and abscisic acid responses, and abscisic acid biosynthesis. Plant Cell. 2011;23(6):2169-83.

\section{Ready to submit your research? Choose BMC and benefit from:}

- fast, convenient online submission

- thorough peer review by experienced researchers in your field

- rapid publication on acceptance

- support for research data, including large and complex data types

- gold Open Access which fosters wider collaboration and increased citations

- maximum visibility for your research: over $100 \mathrm{M}$ website views per year

At BMC, research is always in progress.

Learn more biomedcentral.com/submissions 